\title{
Clasificación de las causas que determinan estadía inadecuada útil para la gestión hospitalaria
}

\author{
Usefulness of the classification of causes of inappropriate \\ lengths of stay for the hospital management
}

\author{
Lic. María Eugenia Elorza,' Lic. Nadia Vanina Ripari,' Lic. Franco Cruciani,"' \\ Dra. Nebel Silvana Moscoso,' Dra. María Eugenia Gullace" \\ I Consejo Nacional de Investigaciones Científicas y Técnicas (CONICET) de Argentina. \\ Universidad Nacional del Sur, Bahía Blanca. Buenos Aires, Argentina. \\ "Universidad Nacional del Sur, Bahía Blanca. Buenos Aires, Argentina.
}

\section{RESUMEN}

Introducción: la búsqueda de eficiencia en la gestión hospitalaria motiva el empleo de indicadores de gestión que detecten y evalúen la presencia de estancias hospitalarias inadecuadas.

Objetivo: diseñar una clasificación de las causas que determinan una estadía hospitalaria inadecuada adaptada al contexto del sistema de salud argentino.

Métodos: se revisaron 25 artículos publicados entre los años 1990 y 2009 en revistas argentinas y de otras nacionalidades, en idioma inglés y español. Para la exploración se incluyeron buscadores genéricos de Internet y las bases de datos bibliográficos: Medline, Cochrane y Lilacs. Las palabras clave empleadas fueron: inappropriateness, inappropriate hospital days, the appropriateness evaluation protocol, factors associated with inappropriate hospitalization days, uso inadecuado de la hospitalización, lenght of stay.

Sintesis de los datos: es necesario distinguir entre ingresos y estadías inadecuadas. Las causas de estas últimas, según la clasificación diseñada, pueden deberse a la responsabilidad de: 1. La planificación y gestión hospitalaria. 2. El médico. 3. La familia del paciente. 4. El sistema de salud.

Conclusiones: el diseño de esta clasificación es una herramienta útil para la implementación de indicadores de gestión hospitalaria que involucren los días de estadía.

Palabras clave: gestión hospitalaria, indicadores, estadía inadecuada. 


\section{ABSTRACT}

I ntroduction: the search for the hospital management efficiency encourages the use of management indicators that detect and assess inappropriate lengths of hospital stay.

Objective: to design a classification of the causes that determine inappropriate lengths of hospital stay, adjusted for the Argentinean health system setting.

Methods: twenty five articles, both in English and Spanish, published from 1990 to 2009 in Argentinean journals and in those of other nations, were reviewed. For this search, the generic Internet's searchers and the main databases such as Medline, Cochrane, Lilacs, and others were consulted. The key words were inappropriateness, inappropriate hospital stays, the appropriateness evaluation protocol, factors associated with inappropriate hospitalization days, uso inadecuado de la hospitalización, length of stay.

Results: it is necessary to differentiate incomes from inappropriate lengths of stay. The designed classification included the following causes: 1. Difficulties in hospital planning and management, 2 . The physician's responsibility, 3. The responsibility of the patient's family, 4 . The responsibility of the health care system.

Conclusions: the design of this classification is a useful tool to implement hospital management indicators involving the length of hospital stay.

Key words: hospital management, hospital management indicators, lengths of hospital stay, inappropriateness.

\section{NTRODUCCI ÓN}

En la última década, Argentina experimentó un aumento sostenido en los precios de los recursos en el sector de la salud que generó un impacto creciente en los costos de las instituciones hospitalarias, públicas y privadas. Este aumento se encuentra asociado, tanto a las innovaciones biomédicas y tecnológicas desarrolladas a nivel mundial como también al efecto de la inflación general de la economía.

Al mismo tiempo, existe una permanente preocupación por el uso ineficiente de los recursos sanitarios que sumado a las restricciones presupuestarias vigentes en el sector, pone de relieve la necesidad de implementar estrategias por parte de los gestores hospitalarios. Si bien este desafío lo enfrentan instituciones públicas y privadas, los hospitales públicos en particular, poseen restricciones adicionales asociadas al tipo de mecanismo de pago utilizado (presupuestos fijos y prospectivos, generalmente anuales), que en circunstancias inflacionarias, ponen en riesgo su capacidad de financiamiento. En este contexto, es relevante la utilización de indicadores de gestión para la toma de decisiones orientados a la optimización en la asignación de los recursos sanitarios.

Actualmente existen diferentes indicadores de gestión hospitalaria que buscan valorar de forma continua las actividades desarrolladas en relación con el cumplimiento de un objetivo determinado. ${ }^{1}$ Diversos autores han realizado clasificaciones alternativas de estos instrumentos. En general, los indicadores brindan información cuantitativa que frecuentemente permiten detectar problemas en la atención hospitalaria. Sin embargo, el análisis de cada uno de estos y sus causas debe ser abordado mediante 
una investigación específica y profunda habitualmente basada en información cualitativa. $^{2}$

En particular, en los servicios de internación, los indicadores de producción ${ }^{3}$ frecuentemente utilizados son:

1. Duración de la estadía (usualmente encontrada en la literatura internacional bajo el nombre de Length of Stay o la sigla LOS) y que se mide como la diferencia en días, entre la fecha de alta y la de ingreso a la institución hospitalaria. ${ }^{4}$

2. Estancia media (estándar o corregida), que mide el número de días de ingreso promedio por paciente egresado del servicio u hospital. ${ }^{1}$

Estos indicadores son útiles para analizar la eficiencia en el uso de los recursos cuando se les compara con los costos incurridos durante el proceso. Sin embargo, para profundizar en el análisis de la eficiencia, es necesario evaluar la adecuación de cada día de estadía en cada paciente. En este sentido, se consideran inadecuados los días de estadía en los que el paciente, aún con necesidad de mantenerse ingresado, podría atenderse en centros asistenciales de diferente complejidad o en menor tiempo, y cuando directamente no debió estar ingresado. ${ }^{4}$ La existencia de esta ineficiencia produce diversos efectos económicos adversos, sea la institución de análisis pública o privada.

En el primer caso, ocasiona la congestión de los servicios poniendo en riesgo su objetivo de maximizar el beneficio social. ${ }^{1,5,6}$ En el caso de los hospitales privados, el efecto dependerá del mecanismo de pago institucional asociado al paciente internado. Si este se encuentra asegurado y el contrato con su seguro de salud establece que se paga por prestación, entonces el hospital traslada el costo total al pagador ya que cobrará por todos los días de ingreso independientemente de su adecuación. Sin embargo, si el mecanismo de pago es per cápita*, la institución asume los costos totales del ingreso y recibe por el servicio brindado el valor de una tarifa fija (sin ajuste por riesgo). Por otro lado, si no existe un tercer pagador y es el paciente quien debe afrontar el pago de su ingreso mediante gasto de bolsillo, los costos totales serán absorbidos mediante su nivel de riqueza.

En general, el análisis de la adecuación de la estadía puede ser útil en el diseño de estrategias tendientes a reducir los días de estancia dado que se considera uno de los principales determinantes de los costos hospitalarios directos. ${ }^{7}$ Si bien, en las últimas décadas las investigaciones se centraron en este aspecto, algunas más recientes muestran que esta intervención de gestión no genera necesariamente un aumento en la eficiencia debido a que, si bien un día adicional de ingreso hospitalario genera un aumento en los costos totales, estos crecen de forma decreciente. ${ }^{8}$

Independientemente de estas consideraciones, en el caso de los servicios públicos, cualquier reducción de los costos hospitalarios tendrá importantes implicaciones dado que el objetivo de estas instituciones es maximizar la cantidad de prestaciones (ingresos) sujetas a un presupuesto fijo.

Para diseñar estrategias de corrección del uso inadecuado ${ }^{9}$ de los recursos, se debe avanzar en el diseño de un sistema de información que identifique de forma permanente y apropiada la adecuación de los ingresos. Sin embargo, una tarea previa es reconocer cuales son las causas asociadas a este fenómeno.

El presente trabajo se propone diseñar una clasificación de las causas que determinan una estadía hospitalaria inadecuada adaptada al contexto del sistema de salud argentino. 


\section{MÉTODOS}

Se revisaron 25 artículos publicados entre los años 1990 y 2009 en revistas argentinas y de otras nacionalidades, en idioma inglés y español. Para su localización se incluyeron buscadores genéricos de Internet y las principales bases de datos bibliográficos: Medline, Cochrane, Lilacs, entre otras. Los términos empleados en la búsqueda fueron: inappropriateness, inappropriate hospital days, the appropriateness evaluation protocol, factors associated with inappropriate hospitalization days, uso inadecuado de la hospitalización, lenght of stay (LOS).

\section{SÍ NTESIS DE LOS DATOS}

En la literatura científica se reconoce que una estadía es inadecuada cuando los cuidados prestados en la misma podrían haber sido brindados en un nivel asistencial inferior o en un plazo más breve. En particular, las estadías prolongadas suelen alertar a los responsables de la gestión sobre la presencia de dificultades, lo que se encuentra fuertemente asociado a la evidencia que sugiere que la probabilidad de inadecuación de cualquier día de estancia aumenta con su prolongación. ${ }^{10} \mathrm{Sin}$ embargo, suele ser difícil para los gestores hospitalarios establecer un criterio de adecuación.

Para determinar si una estadía es adecuada es posible analizar la o las causas por las cuales se está produciendo el ingreso hospitalario. Los gestores interesados en el análisis del desempeño hospitalario, disponen de un instrumento que es frecuentemente utilizado para determinar la adecuación de las estancias hospitalarias. ${ }^{11}$ Se trata del Protocolo de Evaluación de la Adecuación, creado en 1981 por Gertmann y Restuccia ${ }^{12}$ para un hospital comunitario en Massachusetts. Esta herramienta cataloga los días de permanencia en una institución hospitalaria como adecuados o inadecuados según distintos parámetros o criterios de adecuación. En particular, se puede emplear para identificar las causas de estancias inadecuadas. Diversos autores han demostrado que es válido y útil en su aplicación en hospitales de agudos, ${ }^{13-15}$ aunque según Peiró y otros, ${ }^{13}$ no está libre de limitaciones. Es utilizado en países de la Comunidad Europea y en Estados Unidos pero existen muy pocos antecedentes de su aplicación en Latinoamérica en general y particularmente en Argentina. ${ }^{16}$

En general, las estadías adecuadas encuentran su causa y justificación en las condiciones biológicas y clínicas de los pacientes (que pueden incluir conductas de riesgo) que se consideran potencialmente inmodificables en el corto plazo. Estas causas incluyen: infecciones de heridas quirúrgicas, edad avanzada, alcoholismo, tabaquismo, alteraciones motrices, afección del sistema nervioso, ingresos en cuidados intensivos, presencia de comorbilidades, entre otras. ${ }^{17-19}$

Al analizar las causas asociadas a las estancias inadecuadas, se observa que pueden ser producidas por dificultades que surgen antes del proceso de internamiento y que determinan la existencia de ingresos inadecuados, ${ }^{20}$ y dificultades que surgen durante el proceso de internamiento y determinan la aparición de estadías inadecuadas.

En relación con los ingresos inadecuados, ${ }^{21-23}$ se destacan los prematuros, motivados por procedimientos diagnósticos o terapéuticos que no se realizan en el tiempo previsto. Un ejemplo de este fenómeno son los ingresos previos a días no laborales como fines de semana, feriados o por vacaciones del personal. ${ }^{17,21-27}$ Carey y otros, ${ }^{17}$ 
observan que se producen con más frecuencia por fines de semana, particularmente los días domingos.

También se puede ocasionar ingresos inadecuados cuando el paciente es internado porque necesita atención institucional pero en un nivel de complejidad distinto. Por ejemplo, pacientes que requieren cuidados permanentes o paliativos pero que deberían ser asistidos en un hospital de crónicos, una clínica psiquiátrica o una residencia de ancianos y no en un hospital de agudos. ${ }^{28}$ En particular, en los hospitales públicos de Argentina, también se registran ingresos inadecuados pero necesarios, en situaciones donde existen sospechas de abuso del paciente, en cuyo caso se le ingresa a la institución por custodia.

Mozes, San Román y otros, ${ }^{10,24}$ observan ingresos inadecuados en pacientes que deben someterse a procedimientos diagnósticos, tratamientos médicos quirúrgicos que podrían realizárseles de forma externa. ${ }^{10,16,20,21,23,24}$ Una situación similar ha sido apreciada por Vitali ${ }^{16}$ en un hospital público de la Argentina, que identifica admisiones inadecuadas debido a que los pacientes tienen domicilios alejados de la institución.

De acuerdo a la revisión de la literatura, las estadías inadecuadas pueden surgir por las siguientes causas: 1 . Dificultades en la planificación y gestión hospitalaria, 2. Responsabilidad del médico, 3. Responsabilidad de la familia del paciente y 4. Responsabilidad del sistema de salud. A continuación se detalla cada una de ellas.

\section{Dificultades en la planificación y gestión hospitalaria}

Para Negro Álvarez, Ferreira y otros, ${ }^{29,30}$ una causa frecuente de inadecuación de las estadías son los problemas de calendario para realizar intervenciones quirúrgicas, pruebas diagnósticas $u$ otros tratamientos. En particular, Carey y otros, ${ }^{17}$ mencionan que las cirugías son los procedimientos que, con mayor frecuencia, causan este tipo de retrasos, los principales motivos son las demoras administrativas, la falta o retraso de instrumental quirúrgico o la falta de disponibilidad de quirófano. ${ }^{29}$

Otros autores observan que las pruebas diagnósticas o interconsultas pendientes de resultados aumentan las estadías inadecuadas dado que introducen demoras en la toma de decisiones terapéuticas o diagnósticas. ${ }^{10,17,20,21,23-25,28,31}$ En particular, Antón y otros, ${ }^{32}$ destacan que estas situaciones se producen con más frecuencia en los servicios de cirugía general por los problemas generados en la programación de pruebas diagnósticas preoperatorias. ${ }^{17,20,22,23,29,31}$

Diferentes autores han detectado otras causas de ingresos inadecuados tales como:

1. La permanencia del paciente en una sala distinta a la apropiada. ${ }^{1}$

2. Procedimientos diagnósticos o tratamientos que podrían realizárseles en la condición de pacientes externos una vez indicada el alta hospitalaria. ${ }^{20,21,23,24}$

3. Fallas en la programación de la frecuencia óptima de rondas médicas (por ejemplo: fallas en el "pase de sala"). ${ }^{33}$

\section{Por responsabilidad del médico}

Entre estas causas es posible mencionar el retraso en el alta médica debido a:

1.Que el alta está planeada pero no existen aún las órdenes escritas. ${ }^{21,23}$

2. Ausencia de la importancia de la necesidad de dar el alta, una vez que se ha conseguido el propósito de la hospitalización. ${ }^{18,20,21,24}$

3. Incorrecto o incompleto seguimiento de los pacientes en el registro médico. ${ }^{18,26}$ 
Se destaca la importancia de la capacidad, habilidad y experiencia del médico, así como el uso de buenas guías clínicas para evitar estadías hospitalarias inadecuadas. En particular, se ha detectado que los retrasos en la toma de decisión médica y la incapacidad de establecer el diagnóstico correcto, son causas importante de inadecuación. ${ }^{17-20,23-25,34}$ No menos importante es la diferencia de formación existente entre las distintas especialidades médicas. ${ }^{35}$

\section{Por responsabilidad de la familia del paciente}

En este aspecto se destaca la insuficiente o nula preparación de la familia para la atención del paciente en su domicilio, ${ }^{31,36}$ así como el rechazo por parte de ambos al ingreso en instituciones alternativas. ${ }^{6,20,23,25,26}$ En general, por un lado, los familiares se rehúsan a aceptar el traslado a un hogar de ancianos o clínica psiquiátrica y por otro, puede suceder que la familia no disponga de los recursos suficientes para atender al paciente, siendo su condición socioeconómica la causa de la estancia hospitalaria. Hakim y otros, ${ }^{33}$ señalan la demora en la adaptación de viviendas como causa frecuente que retrasa el alta del paciente.

\section{Por responsabilidad del sistema de salud}

Entre estas causas se observa la falta de disponibilidad de cuidados sanitarios en el domicilio, 6,35,36 o la imposibilidad de derivar al paciente por inexistencia o falta de disponibilidad de plazas en instituciones alternativas, ${ }^{20,23,26,30,33}$ como hogares de ancianos, hospitales para enfermedades crónicas, servicios de atención primaria o servicios de atención domiciliaria. ${ }^{16,17,23,26,31,33,36,37}$

Para otros autores, el tiempo de estadía hospitalaria se prolonga de forma inadecuada por las demoras en la autorización por parte de los seguros de salud (en Argentina llamados "obras sociales" o "empresas de medicina prepaga") de ciertas prestaciones como procedimientos diagnósticos, médicos, quirúrgicos o traslados, ${ }^{31,33}$ o insumos de procedimientos. ${ }^{33}$ Además, se mencionan las fallas en la disponibilidad de servicios de asistencia social en el momento del alta del paciente. ${ }^{19,29,38}$

Diferentes autores analizan los porcentajes que representan en los servicios de internamiento, los ingresos y las estadías inadecuados. Para Angelillo y otros, ${ }^{39}$ el $14,2 \%$ de todas las admisiones hospitalarias se pueden considerar inadecuadas, mientras que las estadías con estas características representan el 37,7\% del total de los días de ingreso hospitalario. En la literatura se observan importantes variaciones en los porcentajes de ingresos y estadías inadecuadas de acuerdo a las metodologías empleadas y las enfermedades analizadas, sin embargo, el porcentaje de inadecuación siempre fue superior al $14 \%$.

\section{CONSI DERACI ONES FI NALES}

Actualmente, el aumento sostenido de los costos en las instituciones hospitalarias sumado a las restricciones presupuestarias existentes en el sector de la salud, motiva el empleo de indicadores de gestión sanitaria. En este sentido, el análisis de la adecuación de los días de estadía es una estrategia valiosa dado que provee información adicional a la suministrada por los indicadores de desempeño del servicio 
y colaborar así en la reducción de los costos hospitalarios y en la optimización de la asignación de los recursos en el sector de la salud.

La revisión de la literatura sugiere que los ingresos inadecuados son un problema recurrente en diferentes servicios hospitalarios mundiales. Las causas señaladas por los autores se relacionan con los diversos actores involucrados en el sistema de atención de la salud: aseguradoras, servicios sociales, profesionales médicos e instituciones hospitalarias o sus gestores, como también el propio paciente y su familia.

Si bien los documentos revisados han analizado críticamente el fenómeno de las causas de ingresos inadecuados, se observa como principal debilidad, que no se emplea la búsqueda y apreciación de las revisiones sistemáticas como metodología de investigación. En este sentido, implementar este tipo de herramienta es la motivación de nuestra futura investigación.

Avanzar en el reconocimiento de las causas de inadecuación de los ingresos hospitalarios, es el primer paso para diseñar e implementar políticas que minimicen este problema y mejoren la eficiencia en la asignación y uso de los recursos. Se reconoce que las potenciales medidas a adoptar tendrán incumbencias en diferentes servicios hospitalarios y actores del sistema de salud dado que, de acuerdo a los resultados obtenidos, las causas podrán ser de tipo administrativas, médicas, sociales o individuales.

Se espera que la clasificación presentada en este trabajo sea un insumo para las instituciones hospitalarias de Latinoamérica y Argentina que se propongan diseñar sistemas de información que registren y cuantifiquen los días de estadía inadecuados. Minimizar este problema en los hospitales públicos en particular, permitirá aumentar la eficiencia redundando en mayores recursos físicos y humanos para la atención de la salud de pacientes sin seguro de salud.

\section{REFERENCI AS BI BLI OGRÁFICAS}

1. Chirinos E, Rivero E, Goyo A, Méndez E, Figueredo C. (2008). Indicadores de Gestión para medir la Eficiencia Hospitalaria. Revista Científica Electrónica Ciencias Gerenciales [Internet]. 2008 [citado 5 Feb 2012]; 10(4):50-63. Disponible en: http://dialnet.unirioja.es/servlet/fichero articulo?codigo $=2875302$

2. Jimenez Paneque RE. Indicadores de calidad y eficiencia de los servicios hospitalarios: una mirada actual. Rev Cubana Salud Pública [Internet]. 2004 [citado 5 Mar 2012]; 30(1). Disponible en: http://scielo.sld.cu/scielo. php?pid=S0864$34662004000100004 \&$ script $=$ sci arttext\&tIng=pt

3. Pina V, Torres L. Indicadores de "output" para el análisis de eficiencia de las entidades no lucrativas en el sector público español. Rev Española Financiación Contabilidad [Internet]. 1995 [citado 5 Abr 2012];24(85): 969-89. Disponible en: http://dialnet.unirioja.es/servlet/dcfichero_articulo?codigo $=44192$

4. Carrasquer Moya C, Solé J over A, Peiró S. Demora en la realización de pruebas diagnósticas y estancias innecesarias en el cáncer de pulmón. Rev Clín Esp [Internet]. 2001[ citado 28 Mar 2012];201:619-26. Disponible en: http://www.elsevier.es/sites/default/files/elsevier/pdf/65 165v201n11a13021066pdf001.pdf

http://scielo.sld.cu 
5. Saleta Canosa JL, Rodriguez Sotillo A, Aboal Seijas A. Versión pediátrica del protocolo de evaluación de la adecuación (pae): aplicación a los cuatro grupos diagnósticos relacionados más frecuentes en un hospital infantil de la Coruña. Rev Esp Salud Pública [Internet]. 1997 [citado 20 Dic 2011];71(3):249-55. Disponible en: http://scielo. isciii.es/scielo.php?script=sci_arttext\&pid=S1135$\underline{57271997000300004 \& \operatorname{lng}=\mathrm{es}}$

6. Vargas González V, Hernández C.2009 Sistemas de Información de costos para la gestión hospitalaria. Rev Ciencias Sociales [Internet]. 2009 [citado 11 Nov 2011]; 15(4):716-26. Disponible en:

http://redalyc. uaemex.mx/pdf/280/28012285012.pdf

7. Saxena SK, Ng TP, Yong D, Fong NP, Gerald K. Total direct cost, length of hospital stay, institutional discharges and their determinants from rehabilitation settings in stroke patients. Acta Neurol Scand. 2006; 114(5):307-14.

8. Meneu R. La revisión de la efectividad de las revisiones de utilización inadecuada. Más medición que adopción de medidas. Rev Calidad Asistencial. 2008;23(5):197-8.

9. Peiró MS, Portella E. Identificación del uso inapropiado de la hospitalización: la búsqueda de la eficiencia. Med Clin (Barc). 1994; 103:65-71.

10. San Román J, Luquero F, de la Fuente L, Pérez-Rubio A, Tamames S, FernándezAvilés $F$, et al. Evaluación de las estancias inadecuadas en un servicio de cardiología. Rev Esp Cardiol[Internet]. 2009 [citado 4 Abr 2012];62(2):211-5. Disponible en: http://www. revespcardiol.org/es/revistas / revista-espa\% C3\%B1ola-cardiologia25/evaluacion-las-estancias-inadecuadas -un-servicio-cardiologia-13132054comunicaciones-breves-2009

11. Pérez-Rubio A, Santos S, Luquero FJ, Tamames S, Cantón B, Castrodeza JJ . Evaluación de la adecuación de las estancias en un hospital de tercer nivel. An Sist Sanit Navar [Internet]. 2007 [citado 18 Oct 2010];30:29-36. Disponible en: http://scielo.isciii.es/scielo.php?pid=S1137-66272007000100003\&script $=$ sci_arttext

12. Gertmann PM, Restuccia JD. The appropiateness evaluation protocol: a technique for assesing unnecessary days of hospital care. Med Care. 1981;19:855-71.

13. Peiró S, Meneu R, Roselló ML, Portella E, Carbonell-Sanchís R, Fernández C. Validez del protocolo de evaluación del uso inapropiado de la hospitalización. Med Clin. 1996; 107: 124-9.

14. Fuente D, Peiró S, Portella E, Marchan C, Aymerich S. Utilización innecesaria de la hospitalización: importancia de la gestión a nivel de Servicio. Rev Calidad Asistencial. 1994; 1: 8-16.

15. Strumwasser I, Paranjpe NV, Ronis DL, Share D, SellL J. Reliability and validity of utilization review crite. Appropriateness Evaluation Protocol, standardized medreviewinstrument, and intensity-severity-discharge criteria. Med Care. 1990; 28: 95-111.

16. Vitali FD. Evaluación de la utilización hospitalaria en un hospital público de la República Argentina. XXIII Jornadas de economía para la salud [Internet]. Cádiz: AES; 2003 [citado 22 Feb 2012]. Disponible en: http://huespedes.cica.es/aliens/jaescadiz/Archivos\% 20pdf/Archivos\%20pdf\% 20tc 1072tc.pdf

http://scielo.sld.cu 
17. Carey MR, Sheth H, Braithwaite RS. 2005 A prospective study of reasons for prolonged hospitalizations on a general medicine teaching service. J Gen Intern Med [Internet]. 2005 [cited 2010 Dic 10]; 20(2): 108-15. Available from: http://www.ncbi.nlm.nih.gov/pmc/articles/PMC1490052/pdf/jgi_40269.pdf

18. Tamames S, Pérez Rubio A, Castrodeza Sanz J, Canton Alvarez MB, Luquero FJ, Santos Sanz S, et al. Factors associated with the appropriate use of preoperatory hospital stays: historical cohort study. BMC Health Serv Res [Internet]. 2007 [cited 2010 Oct 14]; 7(187): 1-6. Available from:

http://www.ncbi.nlm.nih.gov/pmc/articles/PMC2212640/pdf/1472-6963-7-187.pdf

19. Hammond CL, Pinnington LL, Phillips MF. A qualitative examination of inappropriate hospital admissions and lengths of stay. BMC Health Serv Res [Internet]. 2009 [cited 2010 Oct 19]; 9 (44): 1-9. Available from: http://www. biomedcentral.com/content/pdf/1472-6963-9-44.pdf

20. Oterino de la Fuente D, Martínez A, González Fouces I, Peiró S. ¿Es necesario hospitalizar a tantos niños, durante tantos días? La hospitalización innecesaria en pediatría. An Esp Pediatr [Internet]. 1999 [citado 28 Mar 2012];50:373-8. Disponible en: http://www.aeped.es/sites/default/files/anales/50-4 -9.pdf

21. Klar R, Müller U, Mönting JS. Medically inappropriate inpatient care in West Germany. Soz Praventivmed. 1990;35(6):209-12.

22. Schiff E, Modan B, Barzilay Z, Blumstein Z, Fuchs Z, Mozes B. Patterns of unjustified pediatric hospital stay. Isr J Med Sci. 1993; 29(1):33-6.

23. Lorenzo S, Suñol R. An overview of Spanish studies on appropriateness of hospital use. Int J Qual Health Care. 1995; 7(3): 213-8.

24. Mozes B, Schiff E, Modan B. Factors affecting inappropriate hospital stay. Qual Assur Health Care. 199; 3(3):211-7.

25. Chopard P, Perneger TV, Gaspoz JM, Lovis C, Gousset D, Rouillard C, et al. Predictors of inappropriate hospital days in a department of internal medicine. Int J Epidemiol [Internet]. 1998 [cited 2010 Nov 23];27(3):513-9. Availble fom: http://ije. oxfordjournals. org/content/27/3/513. full.pdf

26. Luquero Alcalde FJ, Santos Sanz S, Pérez Rubio A, Tamames Gómez S, Cantón Alvarez MB, Castrodeza Sanz J. Factors determining inappropriate days of stay in a third-level hospital. Gac Sanit [Internet]. 2008 [cited 2012 Mar 28]; 22(1): 48-51.

Available from: http://www.scielosp.org/pdf/gs/v22n1/breve3.pdf

27. Velasco Díaz L, García Ríos S, Oterino de la Fuente D, Suárez García F, Diego Roza S, Fernández Alonso R. Impact on hospital days of care due to unnecessary emergency admissions. Rev Esp Salud Pública [Internet]. 2005 [cited 2010 Sept 17]; 79(5):541-9. Available from: http://www. scielosp.org/scielo.php?pid=S1135$\underline{57272005000500004 \& \text { script }=\text { sci }}$ arttext

28. McDonagh MS, Smith DH, Goddard M. Measuring appropriate use of acute beds. A systematic review of methods and results. Health Policy. 2000;53(3):157-84.

29. Ferreira A, Rígoli F, Bazzani R. Procesos asociados con el tiempo de estadía en el Hospital de Clínicas: estudio en cuatro enfermedades trazadoras. Rev Med Uruguay [Internet]. 1997 [citado 30 May de 2011]; 13:23-36. Disponible en:

http://www.rmu.org.uy/revista/13/1/2/es/4/

30. Negro Álvarez J M, Guerrero Fernández M, Ferrándiz Gomís R. El Protocolo de Evaluación del Uso Inapropiado de la Hospitalización (The Appropriateness Evaluation Protocol) en Alergología. Rev Esp Alergol Inmunol Clín [Internet]. 1998 [citado 9 May 2011]; 3(13):171-80. Disponible en: http://alergomurcia.com/pdf/AEP_1998.pdf 
31. Fellin G, Apolone G, Tampieri A, Bevilacqua L, Meregalli G, Minella C, et al. A. Appropriateness of hospital use: an overview of I talian studies. Int J Qual Health Care. 1995; 7(3):219-5.

32. Antón P, Peiróa S, Aranazc J, Calpenae R, Compañ A, Leutscherg E. Uso inadecuado de la hospitalización en cirugía general. Magnitud, factores asociados y causas. Cir Esp [Internet]. 2005 [citado 9 May 2011]; 78(3): 183-91. Disponible en: http://www.elsevier.es/sites/default/files/elsevier/pdf/36 L36v78n03a13078302pdf001.pdf

33. Hakim EA, Bakheit AM. A study of the factors which influence the length of hospital stay of stroke patients. Clin Rehabil. 1998;12(2):151-6.

34. Cabre M. Factors influencing lenght of hospital stay in community-acquired pneumonia: a study in 27 community hospitals. Epidemiol Infect [Internet]. 2004 [cited 2012 Nov 17]; 132: 82-9. Available from:

http://www.ncbi.nlm.nih.gov/pmc/articles/PMC2870168/pdf/15473144.pdf

35. JGG Panis L, Gooskens M, WSM Verheggen F, Pop P, H Prins M. Predictors of inappropriate hospital stay: a clinical case study. Int J Qual Health Care [Internet]. 2003 [cited 2010 Oct 20]; 15(1):57-65. Available from: http://intahc. oxfordjournals.org/content/15/1/57.full.pdf+html

36. Van Vugt $A B$, Touw CR. Quantification of excessive length of hospital stay ('wrong bed problem'); hip fractures in the elderly. Ned Tijdschr Geneeskd. 1994; 138(36): 1806-10.

37. Paillé-Ricolleau C, Hamidou M, Lombrail P, Moret L. Appropriateness of days of hospitalization: a comparative study at Nantes University Hospital. Press Med. 2009; 38(4): 541-50.

38. Mc Cormick MD. Variation in Length of Hospital Stay in Patients with Communityacquired Pneumonia. Am J Med. 1999; 107:5-12.

39. Angelillo IF, Ricciardi G, Nante N, Boccia A, Bianco A, La Torre G, et al. 2000 Appropriateness of hospital utilization in Italy. Public Health [Internet]. 2000 [cited 2011 May 9]; 114(1): 9-14. Available from:

http://www.publichealth.unisi.it/nante/paper/appropriateness\%200f\%20hospital.pdf

\footnotetext{
* Se paga una cantidad fija por cada persona bajo la responsabilidad del médico por un período determinado. Se paga por estar "disponible para tratar" más que propiamente por "tratar". Es decir, la tarifa se percibe con independencia de que los pacientes utilicen o no los servicios del médico, así como la frecuencia de sus visitas.
}

Recibido: 22 de mayo de 2012.

Aprobado: 29 de junio de 2012.

María Eugenia Elorza. Universidad Nacional del Sur. 12 de Octubre 1198. Piso 2 do. Gabinete de Becarios. Bahía Blanca (Buenos Aires), Argentina. Teléf.: 54-2914595101 interno 2740. Correo electrónico: meugilorz@hotmail.com 